A microfabricated steel and glass radiation detector with inherent wireless signaling

This article has been downloaded from IOPscience. Please scroll down to see the full text article.

2011 J. Micromech. Microeng. 21015003

(http://iopscience.iop.org/0960-1317/21/1/015003)

View the table of contents for this issue, or go to the journal homepage for more

Download details:

IP Address: 141.211.173.82

The article was downloaded on 06/04/2012 at 16:58

Please note that terms and conditions apply. 


\title{
A microfabricated steel and glass radiation detector with inherent wireless signaling
}

\author{
Christine K Eun and Yogesh B Gianchandani \\ Department of Electrical Engineering and Computer Science, University of Michigan, Ann Arbor, MI \\ 48109, USA \\ E-mail: eunc@umich.edu
}

Received 5 September 2010, in final form 5 November 2010

Published 10 December 2010

Online at stacks.iop.org/JMM/21/015003

\begin{abstract}
This paper describes an investigation of the performance compromises imposed by a manufacturing approach that utilizes lithographic micromachining processes to fabricate a wireless beta/gamma radiation detector. The device uses in-package assembly of stainless steel electrodes and glass spacers. These elements are micromachined using photochemical etching and powder blasting, respectively. The detector utilizes a commercial, TO-5 package that is hermetically sealed at 760 Torr with an Ar fill-gas. Gas microdischarges between the electrodes, which are initiated by the radiation, transmit wideband wireless signals. The detector diameter and height are 9 and $9.6 \mathrm{~mm}$, respectively, and it weighs $0.97 \mathrm{~g}$. The device performance has been characterized using various sealed, radioisotope sources, e.g., 30-99 $\mu \mathrm{Ci}$ from ${ }^{137} \mathrm{Cs}$ (which is a beta and gamma emitter) and $0.1 \mu \mathrm{Ci}$ from ${ }^{90} \mathrm{Sr}$ (which is a pure beta emitter). It has a measured output of $>15.5$ counts $\mathrm{s}^{-1}$ when in close proximity to $99 \mu \mathrm{Ci}$ from ${ }^{137} \mathrm{Cs}$. The wireless signaling spans $1.25 \mathrm{GHz}$ at receiving antenna-to-detector distances $>89 \mathrm{~cm}$, when in close proximity to a $0.1 \mu \mathrm{Ci}{ }^{90} \mathrm{Sr}$ source. The estimated intrinsic detection efficiency (i.e. with the background rate subtracted) is $3.34 \%$ as measured with the biasing arrangement described in the paper.
\end{abstract}

(Some figures in this article are in color only in the electronic version)

\section{Introduction}

There is increasing interest in microsystems that can provide real-time, first alert information on the presence of dangerous radioisotopes. Miniaturized wireless detectors can serve applications ranging from monitoring radiation safety levels of nuclear power plants to guarding against illicit trafficking of radioactive chemicals and port screening for homeland security. The miniaturization of wireless, radiation detectors can lead to ultra-portable and reconfigurable sensor network systems $[1,2]$, lower power requirements, and permit the use of lithographic manufacturing to drive down sensor cost. In addition, leveraging existing commercial, hermetic packages can enable cost-effective, fast prototyping and large-scale manufacturing.

Gas-based detectors (e.g., Geiger counters) are often favored for environmental surveillance efforts (e.g., in looking for radiation leaks and inadvertent contamination) [3]. These are relatively simple and robust, and can operate over a large temperature range, and measure a wide range of radiation species and energies. The basic structure of a detector includes two biased electrodes (anode and cathode) enclosed within a gas-filled chamber. Beta particles directly interact with the gas, causing avalanche discharges, with current pulses that register as 'counts'. Past efforts in micro-patterned gas-based radiation detectors have utilized lithographic microfabrication techniques for enhancing the areal density of electrodes and maintaining precision and the accuracy of the interelectrode spacing [4-10]. The majority of these detectors are employed in high-energy physics for particle tracking, medical diagnostics for x-ray imaging, or plasma diagnostics. Under appropriate conditions, electrical discharges caused by detection events can emit broadband radio frequency radiation in the manner of Marconi transmitters [11]. It has been 


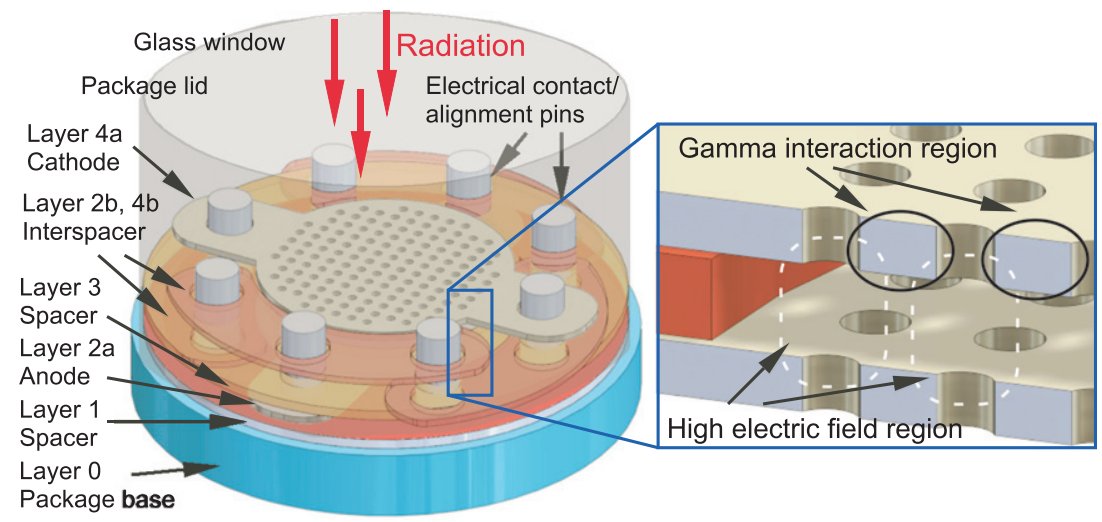

Figure 1. Device concept. The detector comprises a stacked arrangement of stainless steel electrodes (layers $2 \mathrm{a}$, $4 \mathrm{a}$ ) and glass insulators (layers 1,3) assembled within a commercial TO-5 package base. Layer 3 defines the electrode gap spacing. Gamma radiation interacts with the metal layers, which releases photoelectrons into the biased gap. These charged particles trigger an avalanche within the biased gap, leading to wireless signaling.

shown that even microdischarges can be used for wireless signaling [12].

Gas-based detection of gamma radiation relies on converting the photons into photoelectrons and detecting the ejected photoelectrons [13-16]. Detection is often facilitated by using high-density metals for the electrodes and for the walls encapsulating the fill-gas. The use of high-pressure, large atomic number fill-gases [17, 18] can also increase the interaction probability. Gamma radiation interacts with materials in primarily three collision processes, i.e. the photoelectric effect, Compton scattering, and pair production. The energy of the incoming photon determines the collision process. Collisions involving low energy photons are dominated by the photoelectric effect, where the impinging photon transfers all of its energy to an ejected photoelectron. The angle of ejection is determined by the imparted energy. Collisions of higher energy photons (but $E<1.022 \mathrm{MeV}$ ) cause Compton scattering, in which a photoelectron and a secondary photon are emitted. The secondary photon may proceed to participate in further collisions. Finally, for photons with $E>1.022 \mathrm{MeV}$, the collision process is dominated by pair production, where a high-energy electron and positron are ejected. The positron is quickly annihilated by a free electron, resulting in the emission of two photons with energies, $0.51 \mathrm{MeV}$.

In this paper, we investigate the performance compromises imposed by our manufacturing technique that utilizes commercial micromachining processes. More specifically, it involves the fabrication of a miniature detector for beta and gamma radiation that is formed by assembling micromachined steel and glass elements into a commercial TO-5 package $^{1}$. Section 2 describes the device structure and operation, whereas section 3 details the fabrication processes for the metal and glass elements and outlines the assembly procedure. Section 4 describes the experimental results, which include both wireline measurements of terminal currents and wireless measurements of the transmitted spectrum created

${ }^{1}$ Portions of this work have been reported in the conference abstract form in [19]. by the microdischarges. The impact of environment on the received wireless signal is described.

\section{Basic device concepts and operation}

The detector structure includes a stacked pair of steel electrodes and insulating glass elements that are hermetically packaged inside a fill-gas (figure 1). A glass layer insulates the package base from the steel elements (layer 1). The steel elements (layers 2a and 4a) are perforated and separated by a glass spacer (layer 3), which defines the anode-cathode gap spacing. The interspacers are glass elements that lie inplane with the electrode to provide protection against spurious discharges near high-field regions between the steel elements and pins. The package pins serve the additional purpose of spatial alignment of the electrodes during device assembly. During device operation, beta radiation passes through the perforations and directly interacts with the fill-gas, whereas gamma radiation mostly interacts with the steel electrodes to generate photoelectrons. Beta radiation or photoelectrons initiate current-driven avalanche pulses between the biased electrodes, which transmit wideband wireless signals.

The perforated edges serve as concentrated high field regions used for avalanche amplification of the microdischarge. Electric field strengths on the order of $1-2 \mathrm{MV} \mathrm{m}^{-1}$ (in air at 760 Torr) are necessary for the avalanche mechanism to occur [3]. Finite element analysis (FEA) of the electrode configuration shows that with a $150 \mu \mathrm{m}$ gap spacing and a bias level of $650 \mathrm{~V}$, an electric field between 2 and $3 \mathrm{MV} \mathrm{m}^{-1}$ is generated (figure 2). The analysis predicts high field regions concentrated near the perforated edges and lower field regions near the center of the perforations, as expected. This FEA was performed with COMSOL 3.5a using element type: 'triangular'.

The detection of shielded radiation sources is an important consideration from the viewpoints of homeland security and health care. Assuming that the gamma source forms a narrow beam of photons, and this beam passes through an absorber 
(a)
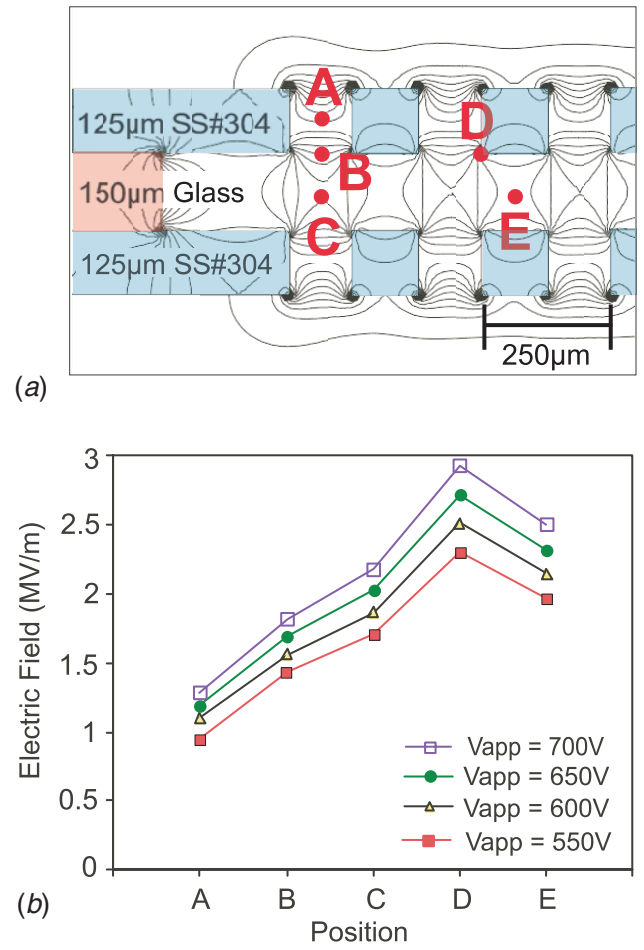

Figure 2. Electric field modeling using COMSOL 3.5a. (a) A cross-section of the 2D model. (b) The generated electric fields range from $1-2 \mathrm{MV} \mathrm{m}^{-1}$ (i.e. near the center of the perforations) to 2-3 $\mathrm{MV} \mathrm{m}^{-1}$ (i.e. near the edges of the perforations). The high-field regions $\left(>1 \mathrm{MV} \mathrm{m}^{-1}\right)$ lead to electron avalanches and microdischarges.

material and hits the detector, the intensity of the gamma radiation is described by [3]

$$
I=I_{0} B\left(t, E_{\gamma}\right) \mathrm{e}^{-\mu t} \quad \text { (photons) }
$$

where $I$ is the number of transmitted photons, $I_{0}$ is the original number of photons, $\mu$ is the linear attenuation coefficient $\left(\mathrm{cm}^{-1}\right)$ of the absorber, and $t(\mathrm{~cm})$ is the thickness of the absorber material. Figure 3 shows the attenuation coefficient as a function of photon energy for lead. For lead, the collision processes are dominated by the photoelectric effect and Compton scattering. For $E_{\gamma}=0.662 \mathrm{MeV}, \mathrm{Pb}$ has a linear attenuation coefficient, $\mu=1.24 \mathrm{~cm}^{-1}$ [20]. The buildup factor, $B$, corrects for the fraction of scattered gamma rays that are retained in the photon beam [3]. It has been calculated, validated, and tabulated by investigators using various numerical methods (e.g., the moment method, the Monte Carlo, integral transport, and discrete ordinate methods) $[21,22]$. The magnitude of the buildup factor depends on the absorber material, thickness, and photon energy. Typical values for $\mathrm{Pb}$ range from 1.2 to 10 [22].

The intrinsic detector efficiency, $\varepsilon_{\text {int }}$, is defined as the percentage of radiation incident on the detector that results in recorded pulses [3]. Assuming a point isotropic source of activity, $A(\mathrm{Ci})$, a source-detector distance, $d_{1}(\mathrm{~m})$, and a detector surface area (i.e. facing the direction of the source), $S_{D}\left(\mathrm{~m}^{2}\right)$, then $\varepsilon_{\text {int }}$ can be rewritten as

$$
\varepsilon_{\text {int }}=\frac{\text { Measured count rate }(\mathrm{cps})}{A \cdot 3.7 \times 10^{10} \cdot \frac{S_{D}}{4 \pi \cdot d_{1}^{2}}} \times 100 .
$$

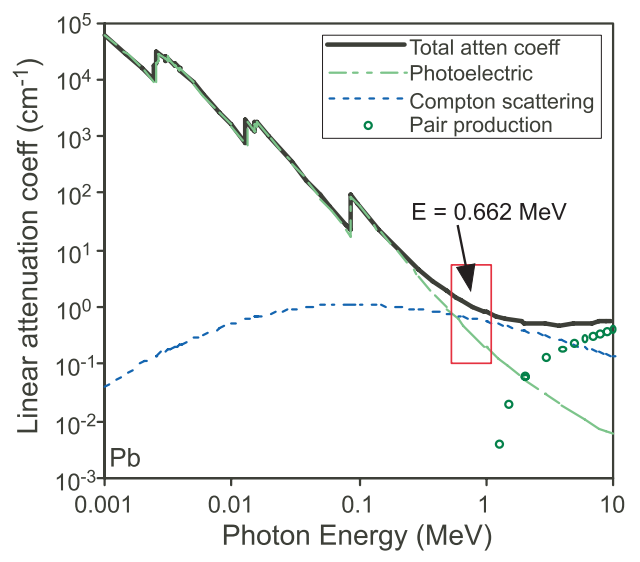

Figure 3. Linear attenuation coefficient, $\mu$, for $\mathrm{Pb}$ as a function of photon energy. For $0.662 \mathrm{MeV}$ photons, $\mu=1.24 \mathrm{~cm}^{-1}$. The dominant scattering mechanisms for ${ }^{137} \mathrm{Cs}$ photons are photoelectric and Compton scattering.

\section{Device fabrication and assembly}

All of the detector elements are manufactured by commercial processes. In particular, the electrodes are formed by photochemical machining of steel foil, whereas the glass spacers are formed by powder blasting. To fabricate the electrodes (figure 4(a)), dry photoresist is laminated on $125 \mu \mathrm{m}$ thick stainless steel (\#304). Following doublesided lithography, the sample is through-etched by a hot etchant spray. In this particular design, $125 \mu \mathrm{m}$ diameter circular perforations are formed, with $250 \mu \mathrm{m}$ center-tocenter spacing. The glass spacer elements are fabricated using a micro-abrasive jet process (figure $4(b)$ ). A protective masking layer is lithographically patterned on a $150 \mu \mathrm{m}$ thick borosilicate glass substrate. An abrasive powder mixed with compressed air is sprayed onto the surface, chiseling the desired pattern. The interspacers are machined similarly. A $22^{\circ}$ sidewall results from this process. To facilitate assembly, each element (steel or glass) is designed with alignment openings to line up with the package pins.

The package is an 8-pin TO-5 metal package of $9 \mathrm{~mm}$ diameter and $9.6 \mathrm{~mm}$ height. It comprises a nickel base and a lid composed of a Kovar body with a $750 \mu \mathrm{m}$ thick glass (Corning 7052) window. Electrical feedthroughs are present as insulated pins that perforate the package base. First, a glass spacer is assembled for electrical insulation (figure 5(a)), followed by the anode that is flanked by a pair of interspacers (figure 5(b)). Next, another glass spacer is installed, defining the electrode gap spacing, followed by the cathode that is flanked by a pair of interspacers (figure 5(c)). After assembly, the package is hermetically sealed at 760 Torr with an Ar fillgas, using a commercial resistance projection welding process (figure $5(d)$ ). The overall device weighs $0.97 \mathrm{~g}$.

\section{Experimental results}

The goal of these experiments was to investigate the device capability and identify performance compromises imposed by our manufacturing approach, i.e. leveraging commercial micromachining processes. 
1. Laminate metal with dry PR \& expose

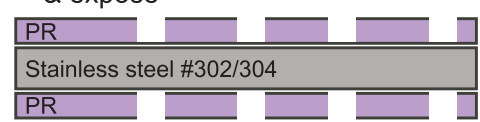

2. Spray heated chemical etchant to remove metal

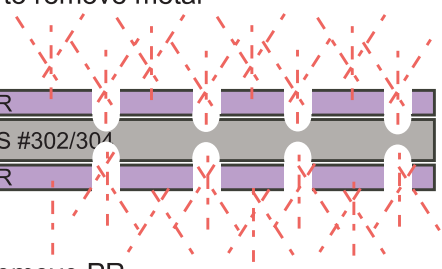

3. Remove PR

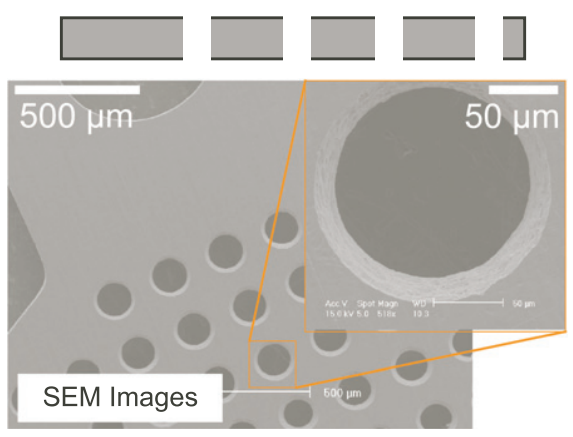

(a)

- $\quad$ Lithographically pattern mask

- Spray glass with abrasive powder

Compressed air Micro-abrasive particles

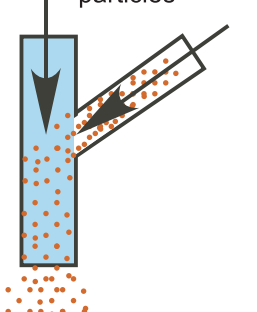

Protective mask

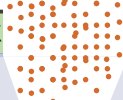

Borosilicate glass

(b)

Figure 4. Fabrication process for $(a)$ photochemically etched electrodes and $(b)$ powder-blasted glass spacers. The SEM images of the electrodes show relatively uniform surface roughness near the $125 \mu \mathrm{m}$ diameter etched perforations.

\subsection{Experimental setup}

The hermetically sealed detector was operated using simple bias circuits (figure 6). The fill-gas was Ar at 760 Torr. The device was characterized with sealed sources of ${ }^{137} \mathrm{Cs}$ using two source strengths, 30 and $99 \mu \mathrm{Ci}$. The source was positioned at various distances, $d_{1}$, from the detector. The detector was operated using two output modes, wireline and wireless. The wireline data were obtained using one of two measurement configurations. The first used an inductive current probe (Tektronix, CT-1) attached to an oscilloscope (Agilent DSO8064A) to obtain count rate data for various
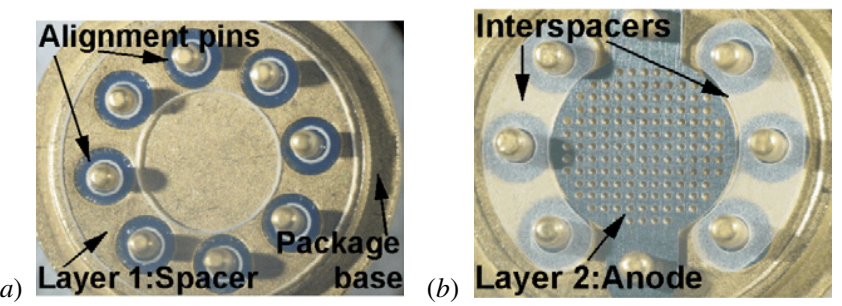

(c)
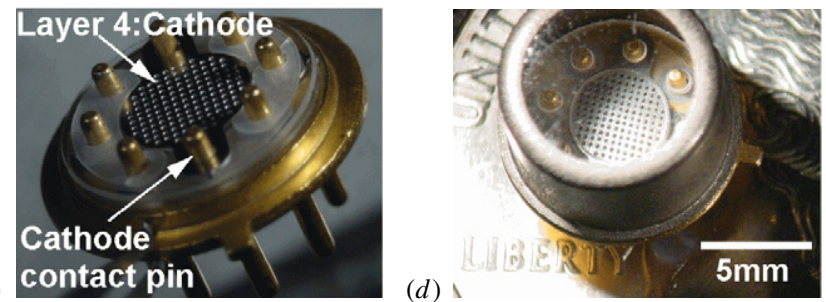

Figure 5. Microassembly of the detector. (a) A glass insulator is positioned onto the base using the package pins for alignment support, $(b)$ followed by the anode and interspacers. Here, the package pins are used for electrical feedthroughs as well as for positioning. (c) Next, the electrode gap defining spacer and then the cathode and interspacers are positioned. $(d)$ The hermetically sealed device encapsulated with 760 Torr of Ar.

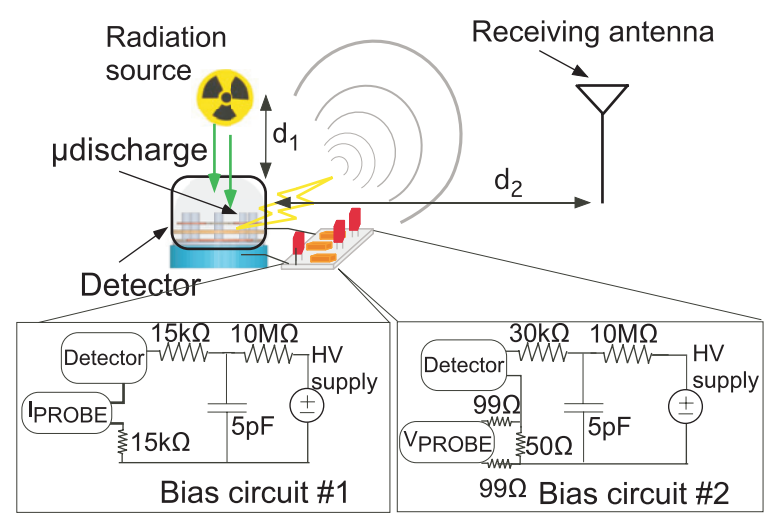

Figure 6. The experimental setup included the packaged device and external bias circuitry positioned at various distances $\left(d_{1}\right)$ from the radiation source. Wireline measurements of the current pulses were taken at the cathode with either bias circuit \#1 or \#2. The $99 \Omega$ resistors in circuit \#2 serve to protect the voltage probe. Wireless measurements were taken with a receiving antenna positioned at various distances $\left(d_{2}\right)$ from the detector. The antenna was attached to a spectrum analyzer or an oscilloscope.

testing parameters (bias circuit \#1). The second employed a high-frequency voltage probe (Agilent, E2678A InfiniMax $3.5 \mathrm{GHz}$ ) attached to a wide bandwidth oscilloscope (Agilent DSA90254A, $2.5 \mathrm{GHz}, 20 \mathrm{GSa} \mathrm{s}^{-1}$ ) in order to more accurately capture the high-frequency characteristics of pulses exiting the detector cathode. It was necessary to slightly modify the bias circuit in order to match the $50 \Omega$ impedance of the probe (bias circuit \#2).

The wireless measurements used a receiving antenna positioned at a distance, $d_{2}$, from the detector and attached to a spectrum analyzer (HP 8563E) or an oscilloscope. The wireless measurements were evaluated in two locations, inside an anechoic chamber in order to accurately capture the RF 


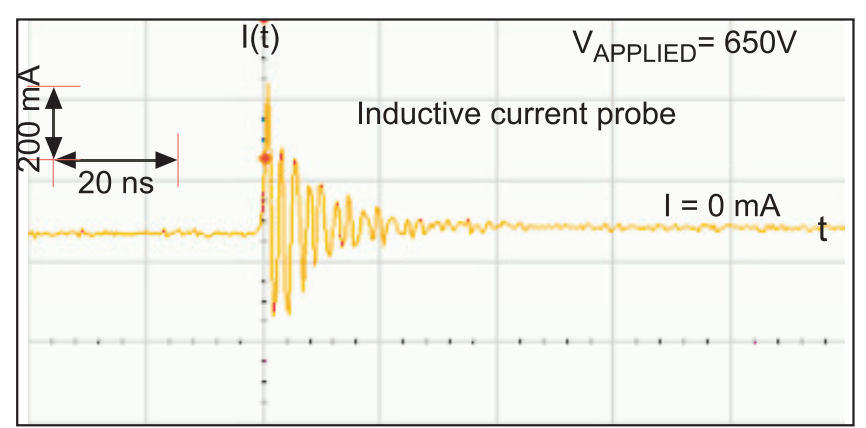

(a)

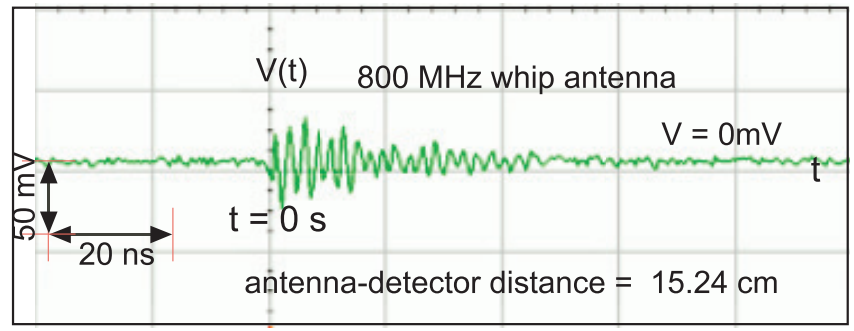

(b)

Figure 7. Wireline and wireless RF monitoring. (a) Current pulse measurement (of a 'count') using a high-frequency inductive current probe attached to the cathode showed approximately $200 \mathrm{~mA}$ peaks and 50-100 ns duration. (b) Transmitted wireless signal received using an $800 \mathrm{MHz}$ whip antenna attached to an oscilloscope. The time domain behavior of the RF transmission followed closely with the current pulse measurement. $0.1 \mu \mathrm{Ci}$ from ${ }^{90} \mathrm{Sr}$ was used.

signal and inside a common laboratory space in order to gauge the signaling capability in a noisy environment.

\subsection{Wireline measurements}

Experiments were performed to determine the impact of several operating conditions on the detector performance, including background radiation, applied bias, device position relative to a stationary and transient radiation source, and shielding on the detector performance. The intrinsic detection efficiencies and receiver operating characteristics (ROC) were also evaluated. The microdischarge current pulses (using bias circuit \#1) were observed to have 50-100 ns duration and $200 \mathrm{~mA}$ peaks (figure 7(a)). Each pulse was registered as a 'count'. The measured background rates (i.e. in the absence of the radiation source) ranged from 0 to $4 \mathrm{cps}$, depending on the bias voltage. (Background count rates can be triggered by cosmic rays or naturally occurring background radiation.)

4.2.1. Impact of an applied bias. The impact of an applied bias on the counting rates was evaluated using a $99 \mu \mathrm{Ci}$ source of ${ }^{137} \mathrm{Cs}$ that was positioned $10 \mathrm{~cm}$ from the detector. A bias (ranging from 630 to $648 \mathrm{~V}$ in $5 \mathrm{~V}$ increments) was applied to the detector and the total counts measured in 30 s intervals. The measured count rates increased with an applied bias (figure 8). The count rates were normalized to $15.5 \mathrm{cps}$, which was the peak count rate measured with an applied bias of $648 \mathrm{~V}$. The

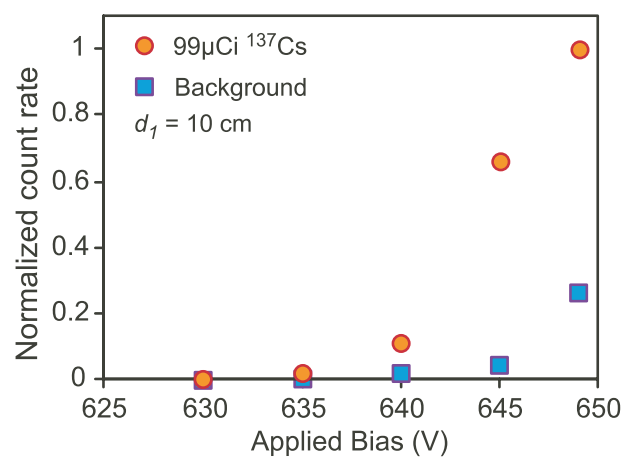

Figure 8. Impact of an applied bias. As the operating voltage increased from 630 to $648 \mathrm{~V}$ in $5 \mathrm{~V}$ increments, the measured count rates with the source present as well as the background rates increased. At $648 \mathrm{~V}$, the peak count rates were 15.5 and $4 \mathrm{cps}$, with and without the source, respectively. The ${ }^{137} \mathrm{Cs}$ source $(99 \mu \mathrm{Ci})$ was positioned $10 \mathrm{~cm}$ from the detector.

background rates (i.e. no source present) were also evaluated and a peak background rate of $4 \mathrm{cps}$ was measured with an applied bias of $648 \mathrm{~V}$. The results showed a slower increase with an applied bias, compared to the rates with the source present.

4.2.2. Impact of source positioning. The impact of the position of a stationary source relative to the detector was also evaluated. Measurements were taken with a $30 \mu \mathrm{Ci}$ source of ${ }^{137} \mathrm{Cs}$ that was separated either axially or laterally from the device by a distance, $d_{1}$. Count rates decreased with increasing $d_{1}$ with a peak count rate of $138 \mathrm{cpm}$ at $d_{1}=0 \mathrm{~cm}$ (figure $9(a)$ ). The detector had somewhat greater sensitivity along its axis of symmetry, providing higher count rates than with a lateral separation of equal distance.

4.2.3. Impact of source shielding. The impact of shielding on detection capability was evaluated using various thicknesses of lead plates (e.g., up to $2 \mathrm{~cm}$ thick attenuators) positioned between a $99 \mu \mathrm{Ci}{ }^{137} \mathrm{Cs}$ source and the detector. As shown in the inset of figure $9(b)$, the lead plates were stacked in contact with the source; the detector was placed $1 \mathrm{~cm}$ beyond the last lead plate. At a bias of $630 \mathrm{~V}$, the measured count rates decreased exponentially with the lead thickness, $t$, as expected (figure $9(b)$ ). Using equation (1), the theoretical upper and lower bounds of the count rate were estimated as a function of lead thickness. The theoretical lower bound (LB) assumed photoelectric interaction with one photoelectron produced per gamma ray and was normalized to fit the peak experimental count rate (i.e. without an attenuator). The theoretical upper bound (UB) assumed Compton scattering with two photoelectrons produced per incident gamma ray (i.e. twice the LB curve). The UB and LB curves were similar in behavior to the measured data.

4.2.4. Beta versus gamma detection. When ${ }^{137}$ Cs decays, it emits beta particles (with an endpoint energy of $0.514 \mathrm{MeV}$ ) $94.4 \%$ of the time and gamma rays (with an energy of $0.662 \mathrm{MeV}) 85.1 \%$ of the time [25]. A $1.25 \mathrm{~cm}$ thick glass 


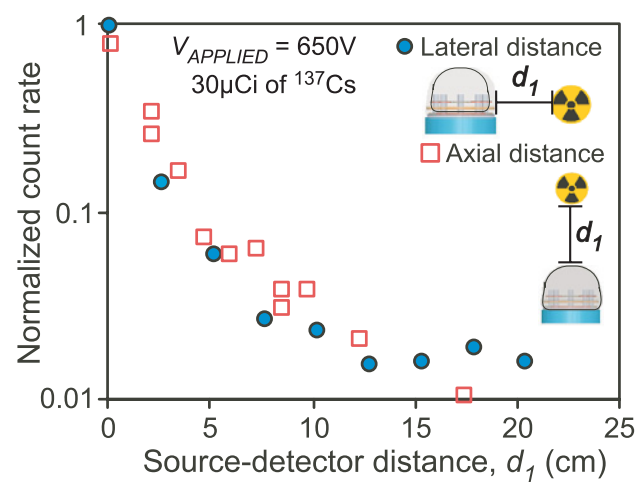

(a)

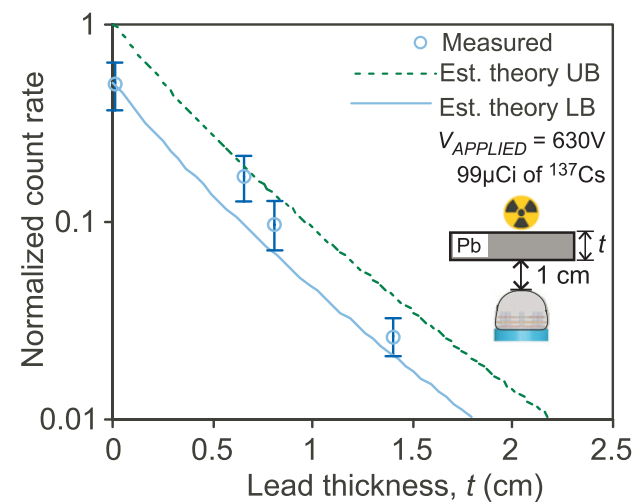

(b)

Figure 9. (a) Impact of source positioning. The measured count rates with the source-detector axially aligned were slightly higher than the count rates with the source-detector laterally aligned. The highest measured count rate was $138 \mathrm{cpm}$ at $d_{1}=0 \mathrm{~cm}$. The applied bias was $650 \mathrm{~V}$ and the background count rate was $1.3 \mathrm{cpm}$. (b) Lead shielding. The measured count rates decreased exponentially with lead thickness, $t$. Error bars indicate one standard deviation from the mean. Taking into account buildup factor, attenuation factor, and solid angle, the theoretical estimate of the counting rate was similar in trend to the measured data. The theoretical upper bound (UB) assumed Compton scattering with two photoelectrons produced per incident gamma ray, while the theoretical. lower bound (LB) assumed photoelectric interactions with one photoelectron per gamma ray. The applied bias was $630 \mathrm{~V}$ and the background count rate was 0.7 cpm. The fill-gas was Ar at 760 Torr.

Table 1. Calculated values for beta and gamma attenuation through a glass shield for ${ }^{137} \mathrm{Cs}$.

\begin{tabular}{ll}
\hline Source activity & $30 \mu \mathrm{Ci}$ \\
Beta energy & $0.512 \mathrm{MeV}$ \\
Thickness of the glass shield & $1.25 \mathrm{~cm}$ \\
Beta energy loss through the shield & $4.76 \mathrm{MeV}$ \\
Thickness of the lid & $760 \mu \mathrm{m}$ \\
Beta energy loss through the lid & $0.290 \mathrm{MeV}$ \\
Total stopping power of glass & $1.71 \mathrm{MeV} \mathrm{cm}^{2} \mathrm{~g}^{-1}$ \\
Density of glass & $2.23 \mathrm{~g} \mathrm{~cm}^{-3}$ \\
Gamma energy & $0.662 \mathrm{MeV}^{\mathrm{a}}$ \\
Linear attenuation coeff. of glass $^{\mathrm{b}}, \mu$ & $0.179 \mathrm{~cm}^{-1}$ \\
Gammas attenuated by the shield & $15-20 \%$ \\
\hline
\end{tabular}

${ }^{a}$ ESTAR, NIST database for $E_{\beta}=0.5 \mathrm{MeV}$ [23]

${ }^{\mathrm{b}}$ XCOM, NIST database for $E \gamma=0.662 \mathrm{MeV}$ [20]

shield was placed between the ${ }^{137} \mathrm{Cs}$ source and the detector in order to block the beta particles (table 1). This is expected to attenuate the gamma count by $15-20 \%$. The measured count rates dropped by $20-35 \%$ with the glass shield present, indicating that the majority of the measured counts were from the detection of gamma radiation. The source-detector distance, $d_{1}$, was $2.1 \mathrm{~cm}$.

4.2.5. Intrinsic detection efficiency. The intrinsic detection efficiency was calculated using the peak count rate of $15.5 \mathrm{cps}$ with a $99 \mu \mathrm{Ci}$ source at $d_{1}=10 \mathrm{~cm}$. The active detection area, $S_{D}$, was $11.8 \mathrm{~mm}^{2}$. Subtracting the background rate (i.e. $4 \mathrm{cps}$ ) and assuming only gamma detection with each gamma ray triggering only one recorded event, the estimated efficiency was $3.34 \%$.

4.2.6. High-frequency measurements. The high-frequency voltage probe allowed a more accurate capture (i.e. compared to the current probe) of the discharge behavior isolated within the detector structure. There were two main pulse shapes observed, a single peak and a double peak (figure 10). Each single-peaked pulse had two main components: an initial peak of the order of 5-10 ns and a longer decaying peak of the order of $100 \mathrm{~s}$ of ns. The double peaks demonstrated an oscillatory effect, where there were two very fast peaks that were 5-10 ns in duration and opposite in direction, followed by a slower decaying tail.

4.2.7. Receiver operating characteristics. The ROC curve depicts the compromise between successful detection events and false alarm rates [3, 24, 26]. The impact upon the ROC curves of sensor integration time over which detection counts are collected was evaluated. The true positive rate (TPR) is the fraction of true detection events (i.e. when a source is present) above a predetermined threshold of counts. The false positive rate (FPR) is the fraction of detection events above the same threshold without a source present, i.e. background events. Figure 11 shows ROC curves for integration times ranging from 60 to $360 \mathrm{~s}$. The curves were calculated from experimental results using MATLAB 7.11. Figure 11 also compares tabulated threshold values for TPR and FPR. For example, for an integration time of $180 \mathrm{~s}$, the threshold values for TPR $=0.5$ and FPR $=0.5$ were 42 and 23 counts, respectively. This indicates that the number of measured counts with a source present were typically twice the background counts. The most favorable operating points appear to be at FPR values ranging from 0.15 to 0.25 . As integration times increased from 60 to $360 \mathrm{~s}$, the likelihood of a true detection event increased from $35 \%$ to $95 \%$ for a corresponding false positive rate of $20 \%$. The source-detector distance was fixed at $30.5 \mathrm{~cm}$.

4.2.8. Impact of a transient source. The ability of the device to detect a transient source at various speeds was evaluated. 


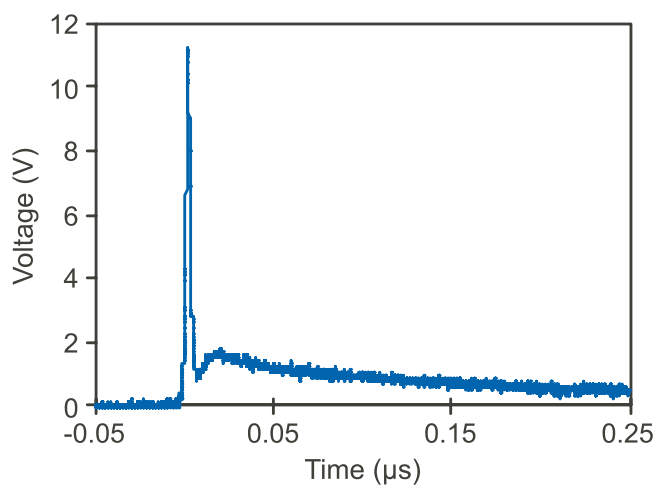

(a)

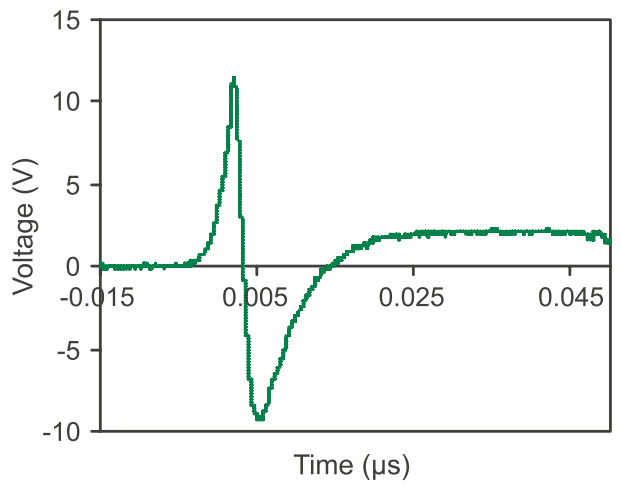

(b)

Figure 10. High-frequency voltage probe measurements showed two basic pulse shapes: $(a)$ a single-peaked pulse, which has two main components, a very fast initial peak (5-10 ns duration) followed by a slower decaying tail (100 s of ns) and (b) a double-peaked pulse, which has two fast peaks, opposite in direction and followed by a slower decaying tail.

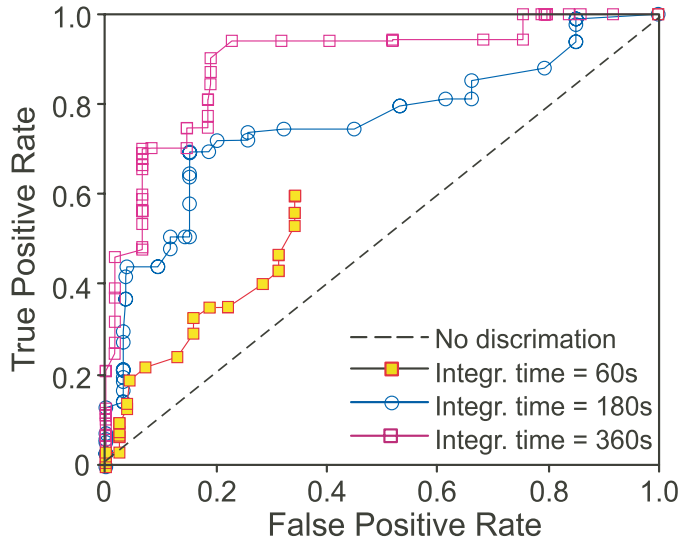

\begin{tabular}{|r|c|c|}
\hline & \multicolumn{2}{|c|}{ Threshold count values (counts) } \\
\hline Integr. time (s) & TPR=0.5 & FPR=0.5 \\
\hline 60 & 10 & $0-1$ \\
\hline 180 & 42 & 23 \\
\hline 360 & 84 & 41 \\
\hline
\end{tabular}

Figure 11. ROC curves (using MATLAB 7.11) for various integration times ranging from 60 to $360 \mathrm{~s}$. Each point represents the tradeoff for a threshold count value. Some threshold count values are listed in the table. The threshold value for TPR $=0.5$ indicates the median of the measured count values with a source present and the threshold for FPR $=0.5$ is the median for the background counts. The ROC improves as the integration time increases due to the increased probability of a detection event. The applied voltage was $790 \mathrm{~V}$ with a fixed source-detector distance of $30.5 \mathrm{~cm}$.

A $99 \mu \mathrm{Ci}{ }^{137} \mathrm{Cs}$ source was translated at a fixed height and velocity along a $30 \mathrm{~cm}$ long straight path, with the detector positioned at a distance of $3.8 \mathrm{~cm}$ orthogonal to the midpoint of the path. An LED attached to the cathode of the detector produced optical pulses to visually indicate each detection event. As the speed of the source increased from 0.03 to $0.5 \mathrm{~m} \mathrm{~s}^{-1}$, the likelihood of signal detection decreased from $94 \%$ to $25 \%$ (figure $12(a)$ ). Negative and positive delays indicate that either an event was detected before or after the source traveled past the detector, respectively (figure 12(b)).
Negative delays were observed predominantly for lower source speeds $\left(0-0.2 \mathrm{~m} \mathrm{~s}^{-1}\right)$, where the device was able to detect the approach of the source. For higher source speeds $\left(0.2-0.5 \mathrm{~m} \mathrm{~s}^{-1}\right)$, an increase in positive delays was observed (as much as $1 \mathrm{~s}$ ). This may be related to the integration time.

\subsection{Wireless measurements}

In order to minimize background noise and reflections and accurately measure the transmitted wireless spectra, the device was operated in an anechoic chamber. The transmitted spectra were measured with a $200 \mathrm{MHz}-1 \mathrm{GHz} \log$-periodic antenna (EMCO 93146) connected to a spectrum analyzer. (An integration period of 1 min was necessary in order to fully capture the transmitted frequency content due to the wide measurement bandwidth and slow scanning speed of the spectrum analyzer.) In the presence of $0.1 \mu \mathrm{Ci}$ from ${ }^{90} \mathrm{Sr}$, the detector transmitted wireless spectra spanning $1.25 \mathrm{GHz}$ with peaks at $550 \mathrm{MHz}, 750 \mathrm{MHz}$, and $1.1 \mathrm{GHz}$ (figure 13). The received signal power decreased with increasing antennadetector distances, $d_{2}$.

The wireless signal from a detector operating in a normal laboratory environment was also evaluated, in order to observe the transient nature and robustness of the signal generation and reception. In this case, an $800 \mathrm{MHz}$ whip antenna $\left(d_{2}=\right.$ $15.25 \mathrm{~cm}$ ) was connected to an oscilloscope. A simultaneous measurement of the time-domain current pulse (using a current probe at the cathode) and the received RF signal showed the instantaneous nature of the transmitted signal and a strong correlation between the antenna response and the microdischarge (figure $7(b)$ ). This experiment demonstrated the ability of the detector to wirelessly transmit count rate information as well as confirmed the ability to receive the RF transmission using simple, off-the-shelf components in a noisy environment.

\section{Discussion and conclusions}

The primary goal of this work was to investigate the performance compromises imposed by our decision to 


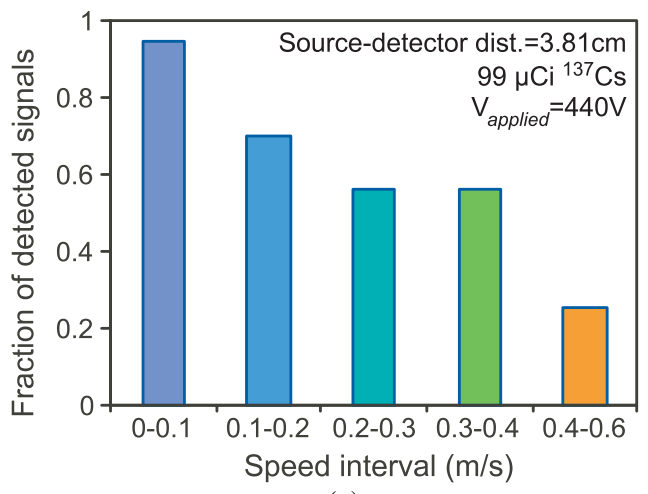

(a)

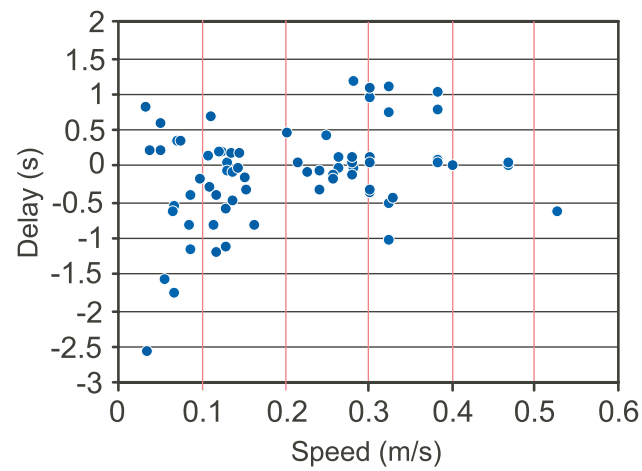

(b)

Figure 12. Transient source detection. (a) As the speed of the source increases, the likelihood of signal detection decreases. For speeds in the range of $0-0.1 \mathrm{~m} \mathrm{~s}^{-1}$, the fraction of detected signals was $94 \%$. The likelihood decreases to near $25 \%$ for speeds in the range of $0.4-0.6 \mathrm{~m} \mathrm{~s}^{-1}$. (b) The measured signal delay as a function of source speed. For lower speeds, there was an increase in negative delays. Negative delays occur when an event is detected prior to the source passing over the detector.

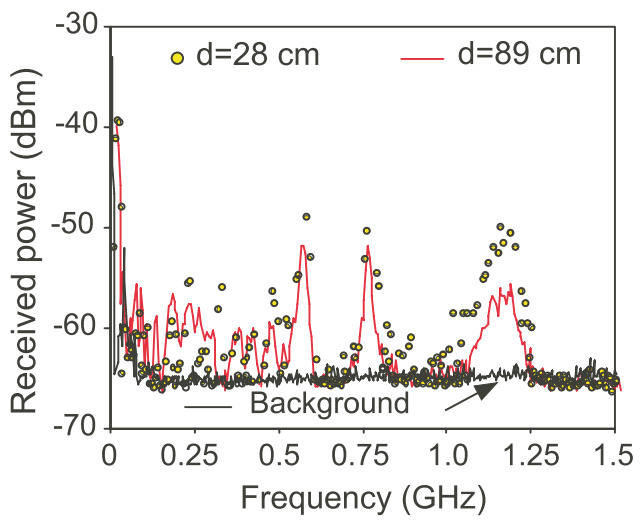

Figure 13. Wireless measurement. The generated wireless spectra measured in an anechoic chamber with a log-periodic antenna attached to a spectrum analyzer showed frequency content spanning $1.25 \mathrm{GHz}$ with peaks at $550 \mathrm{MHz}, 750 \mathrm{MHz}$, and $1.1 \mathrm{GHz}$. $V_{\text {APPLIED }}=650 \mathrm{~V}$. The received wireless power decreased with increasing antenna-detector distance. Strong signaling was observed at distances $>89 \mathrm{~cm}$. $0.1 \mu \mathrm{Ci}$ from ${ }^{90} \mathrm{Sr}$ was used.

leverage commercial micromachining processes. For example, the use of wet etching to machine the electrodes may be a contributing factor to the sensitivity of the detector response to an applied bias. A high-density of structural asperities (of the order of $5-10 \mu \mathrm{m}$ ) has been observed in the sidewalls of the perforations. These sharp features, which are artifacts of the manufacturing process, create localized high-field regions leading to spurious discharges or excessive dependence on bias conditions. Possible approaches to alleviate this sensitivity include electro-polishing of the electrode surface and in particular, targeting the roughness in the sidewalls to decrease the sharp profile. Another approach to reduce the bias dependence and reduce the propensity for spurious discharges is to introduce a trace amount (5-10\%) of a quench agent to the fill-gas (e.g., chlorine or bromine) [3]. Quench gases are a common method to extinguish discharges faster and to reduce spurious discharges, extending electrode lifetimes.

The detector demonstrated two output modes: wireline and wireless. Quantitative assessment of radiation levels is important and can be accomplished by both modes. Using the detector in 'wireline' mode, a quantitative assessment of radiation level is simple; the count rate is proportional to radiation level.

A quantitative assessment of the radiation level while the detector is operating in a 'wireless' mode depends on the duration of the transmitted electromagnetic pulses and the speed of the receiver. As seen in figure 7, the duration of the transmitted signal is $40 \mathrm{~ns}$, which is comparable to the corresponding current pulse duration (20 ns) measured at the cathode. The transmitted wireless signal from the current pulses appears to be nearly instantaneous. Since the FCC allocation of the ultra-wideband (UWB) frequency spectra [27], there has been extensive research in using narrow, impulse-shaped signals (with durations of less than a few ns) to drive low-power, high data rate UWB transceivers that can exceed $110 \mathrm{Mb} \mathrm{s}^{-1}$ [28-32]. Advantages provided by UWB communications include robustness to jamming, as well as a low probability of interception and detection.

Note that for the results presented in figure 13, a spectrum analyzer was used to capture the frequency spectra in order to evaluate the frequency content and bandwidth of the transmitted signal. However, the acquisition speed of the spectrum analyzer is slow and in a single scan some instantaneous wireless content can be missed. Therefore, an integration period of $1 \mathrm{~min}$ was necessary to accurately capture the frequency content while the detector was continuously operated during this period.

As ultimately noted, this is not representative of a dedicated, tuned receiver, which can count individual detection events, particularly when the radiation count is low. Additionally, having a custom-designed receiving antenna tuned to the transmitted frequencies can greatly increase the signal-to-noise ratio (SNR) and improve the detector-toantenna range.

The distance between the sensor and the receiving antenna is limited in this effort because the receiving antenna used is for general purpose and was not designed for this particular application. In addition, the bias circuit for the detector has 
not been optimized for wireless signaling and improvements in this respect can increase the transmitted SNR.

The main goal of the voltage probe measurements (figure 10) was to capture true high-frequency behavior. The ripples that had been observed previously using the inductive current probe were not observed, which suggests that the ripples were artifacts of the probe and not inherent to the current pulse behavior. The majority of the pulses observed were single-peaked clean pulses. Each pulse had a very sharp initial peak (due to the fast mobility of electrons), followed by a slower decaying tail (attributed to the ion mobility). The double-peaked pulses suggest some resonance behavior from the detector, causing the electrons (or ions) to change direction suddenly. Closer observation revealed ripples within the pulse. The oscillations were of the order of $1 \mathrm{~ns}$ in duration, corresponding to a $1 \mathrm{GHz}$ frequency content. This could also be attributed to the resonances of the detector.

The ROC curves offer a graphical representation of compromises for different operating conditions, e.g., the choice of threshold count values. Each point on the curve indicates the TPR and FPR for a particular threshold. As the threshold count value decreases (i.e. by moving to the right of the curve), the likelihood of true positive events and the likelihood of false positives increase. By choosing the appropriate threshold value, a specific TPR and FPR can be achieved. The compromises between integration time and SNR are also evident. Detectors with smaller detection volumes may perform adequately in applications that accommodate larger integration times.

If detection events were uniformly distributed in time, threshold count values would be linear with integration time. However, shorter integration times (e.g., 60 s) indicated a slight nonlinearity, which suggests that the events occurred in clusters or bursts. A further study may provide additional insight into the operational mechanics of the detector and the source detected.

The compromise between integration time and detection capability was also observed for transient sources. As the source speed increases, the corresponding integration time decreases and the rate of detection decreases. The application of the device in this manner demonstrated a simple, threshold detection method for low intensity moving targets.

In this work, a wireless-enabled micromachined radiation detector manufactured using in-package assembly methods has been presented. The overall detector diameter and height were 9 and $9.6 \mathrm{~mm}$, respectively, and it weighed $0.97 \mathrm{~g}$. The device generated count rates $>15.5 \mathrm{cps}$. The estimated intrinsic detection efficiency (i.e. with the background rate subtracted) was $3.34 \%$. The transmitted wideband wireless spectra spanned $>1.25 \mathrm{GHz}$. The main intent of this effort was to explore a configuration that can be easily manufactured and cost-effective. The detector leveraged commercial processes and off-the-shelf packaging components in order to achieve a high-throughput, cost-effective design. This approach to manufacturing and assembly demonstrated a scalable path for increasing detection efficiency for a given form factor. For example, the presented structure can be modified easily to accommodate an arrayed electrode structure, i.e. by simply increasing the number of stacked electrode structures. Miniaturized wireless radiation sensors are envisioned for use in rapidly deployable, mobile network configurations.

\section{Acknowledgments}

This work was supported by the Department of the Army, Micro Autonomous Systems and Technology (MAST) Collaborative Technology Alliance, contract no W911NF-082-0004. The authors thank R Gharpurey for collaboration on the wireless signaling concept, and $\mathrm{K}$ Sarabandi and A Nashashibi for discussions and access to measurement facilities.

\section{References}

[1] Nemzek R J, Dreicer J S, Torney D C and Warnock T T 2004 Distributed sensor networks for detection of mobile radioactive sources IEEE Trans. Nucl. Sci. 51 1693-700

[2] Kyker R, Berry N, Stark D, Nachtigal N and Kershaw C 2004 Hybrid emergency radiation detection (HERD), a wireless sensor network application for consequence management of a radiological release Proc. SPIE 5440 293-304

[3] Knoll G F 2010 Radiation Detection and Measurement 4th edn (New York: Wiley)

[4] Sauli F 2002 Micro-pattern gas detectors Nucl. Instrum. Methods Phys. Res. A 477 1-7

[5] Biagi S F and Jones T J 1995 The microdot gas avalanche chamber: an investigation of new geometries Nucl. Instrum. Methods Phys. Res. A $36172-5$

[6] Oed A 1998 Position-sensitive detector with microstrip anode for electron multiplication with gases Nucl. Instrum. Methods Phys. Res. A 263 351-9

[7] Wada M, Suzuki J and Ozaki Y 1989 Cadmium telluride B-ray detector Sensors and Actuators 19 227-36

[8] Charpak G, Derre J, Giomataris Y and Rebourgeard P 2002 Micromegas, a multipurpose gaseous detector $\mathrm{Nucl}$. Instrum. Methods Phys. Res. A 478 26-36

[9] Nishimura $\mathrm{H}$ et al 2006 The performance of the micro time projection chamber based on $\mu$-PIC 2006 IEEE Nuclear Science Symp. Conf. Record vol 6 pp 3860-4

[10] Son C and Ziaie B 2006 Electret based wireless micro ionizing radiation dosimeter IEEE Int. Conf. on MEMS (January 2006) pp 610-3

[11] Brittain J E 2004 Electrical engineering Hall of Fame: Guglielmo Marconi Proc. IEEE 92 1501-4

[12] Eun C K, Gharpurey R and Gianchandani Y B 2010 Wireless signaling of beta detection using microdischarges J. Microelectromech. Syst. 19 785-93

[13] Shafrir B and Seidman A 1975 High efficiency gamma-ray metal converters Nucl. Instrum. Methods 129 177-86

[14] Shimoni U, Sheinfux B, Seidman A, Grinberg J and Avrahami Z 1974 Investigations on metal converters for gamma-ray detection and mapping Nucl. Instrum. Methods 117 599-603

[15] Nakamura M 1983 The energy spectra and the quantum efficiencies of electrons emitted from the metallic elements irradiated by ${ }^{60}$ Co gamma-rays $J$. Appl. Phys. 54 3141-9

[16] Hirschfelder J O, Magee J L and Hull M H 1948 The penetration of gamma-radiation through thick layers Phys. Rev. 73 852-62

[17] Lehner C E, He Z and Knoll G F 2003 Intelligent gamma-ray spectroscopy using 3-D position-sensitive detectors IEEE Trans. Nucl. Sci. 50 1090-7 
[18] Kiff S D, He Z and Tepper G C 2005 A new coplanar-grid high-pressure xenon gamma-ray spectrometer IEEE Trans. Nucl. Sci. 52 2932-9

[19] Eun C K and Gianchandani Y B 2010 A wireless-enabled radiation detector using micromachined steel and glass elements in a TO-5 package A Solid-State Sensors, Actuators and Microsystems Workshop (June 2010, Hilton Head Island, SC, USA)

[20] Berger M J et al 1998 XCOM: photon cross sections database NIST standard reference database http://physics.nist. gov/PhysRefData/Xcom/Text/XCOM.html

[21] Bureau of Radiological Health and the Training Institute Environmental Control Administration 1970 Radiological Health Handbook (Washington, DC: Government Printing Office)

[22] Shleien B, Slaback L A and Birky B 1992 Handbook of Health Physics and Radiological Health Revised edn (Silver Spring, MD: Scinta, Inc.)

[23] Berger M J, Coursey J S, Zucker M A and Chang J 2005 ESTAR: stopping power and range tables for electrons, protons, and helium ions NIST standard reference database http://physics.nist.gov/PhysRefData/Star/Text/ESTAR.html

[24] Fawcett T 2006 An introduction to ROC analysis Pattern Recognit. Lett. 27 861-74
[25] Firestone R B and Ekstrom L P 1999 LBNL isotopes project LUNDS Universitet, Version 2.1 http://ie.lbl.gov/toi/index.asp

[26] Brown C D and David H T 2006 Receiver operating characteristics curves and related decision measures: a tutorial Chemometr. Intell. Lab. Syst. 80 24-38

[27] FCC 2002 'Revision of Part 15 of the Commission's Rules Regarding Ultra-Wideband Transmission Systems' First Report and Order, FCC 02-48 http://hraunfoss.fcc.gov/ edocs_public/attachment/FCC-02048A1.pdf

[28] Wentzloff D 2007 Pulse-based ultra-wideband transmitters for digital communication Department of Electrical Engineering and Computer Science, MIT

[29] Heydari P 2005 Design considerations for low-power ultra wideband receivers 6th Proc. Int. Symp. on Quality Electronic Design pp 1-6

[30] Namgoong W 2003 A channelized digital ultrawideband receiver IEEE Trans. Wirel. Commun. 2 502-10

[31] Yin H, Wang Z, Ke L and Wang J 2010 Monobit digital receivers: design, performance, and application to impulse radio IEEE Trans. Commun. 58 1695-704

[32] Aiello G R and Rogerson G D 2003 Ultra-wideband wireless systems IEEE Microw. Mag. 4 36-47 